\title{
Pseudo 2- Çaprazlanmış Modüller ve Pseudo 3- Çaprazlanmış Modüller
}

\author{
Sedat PAK1 1 (i) Özgün GÜRMEN ALANSAL 2 (iD Uğur CESUR ${ }^{3}$
}

${ }^{1}$ Necmettin Erbakan Üniversitesi Fen Fakültesi, Matematik ve Bilgisayar Bölümü, Ahmet Keleşoğlu Yerleşkesi, A Blok, Oda No: 110, Meram, Konya, Türkiye (Sorumlu Yazar/Corresponding Author) 2 Dumlupınar Üniversitesi, Fen Edebiyat Fakültesi, Matematik Bölümü, Kütahya, Türkiye ${ }^{3}$ Milli Eğitim Bakanlığı, 42140, Meram, Konya, Turkey

\begin{tabular}{ll}
\hline Makale Bilgileri & ÖZET \\
\hline Makale Geçmişi & Homotopi 2 - tiplerinin cebirsel modelleri üzerindeki çalışmasında J.H.L.Whitehead [1], \\
Geliş: 13.11.2020 & çaprazlanmış modül kavramını ilk olarak gruplar üzerine tanımlamış, sonrasında ise \\
Kabul: 18.12.2020 & çaprazlanmış modül cebirler üzerinde T. Porter [2] tarafından tanımlanmıştır, ayrıntılı bilgi \\
Yayın: 31.12.2020 & için [3,4,5] bakılabilir. Conduché [6] ise 3 - tip homotopi modeli olarak 2-çaprazlanmış model \\
Anahtar Kelimeler: & kavramını tanımlamıştır. Carrasco-Cegarra [7], hiper çaprazlanmış kompleks kavramını \\
Çaprazlanmış Modül, & tanımlamış ve hiper çaprazlanmış kompleksler kategorisinin simplisel gruplar kategorisine \\
Homotopi, Moore & denk olduğunu göstermiştir. Mutlu - Porter [8], simplisel gruplarda Peiffer çiftlerinden \\
kompleks, & faydalanmışlardır. Daha sonra 3- çaprazlanmış modül yapısı, Arvasi, Kuzpınarı ve Uslu [9] \\
Pseudosimplisel grup. & tarafından tanımlanmıştır. Inassaridze [10], pseudosimplisel grup yapısını tanımlamış, Akça \\
& ve Pak [11] pseudo 2- çaprazlanmış modülü tanımlamıştır. Bu çalışmada pseudo 3- \\
& çaprazlanmış modül kavramını tanımlanarak, Arvasi , Kuzpınarı ve Uslu [9] tarafindan \\
& tanımlanan 3-çaprazlanmış modüller ile arasındaki ilişki incelenmiştir ve pseudo 3- \\
& çaprazlanmış modüller kategorisi ile 3-çaprazlanmış modüller kategorisinin denkliği elde \\
& edilmiştir [12].
\end{tabular}

\section{Pseudo 2- Crossed Modules and Pseudo 3- Crossed Modules}

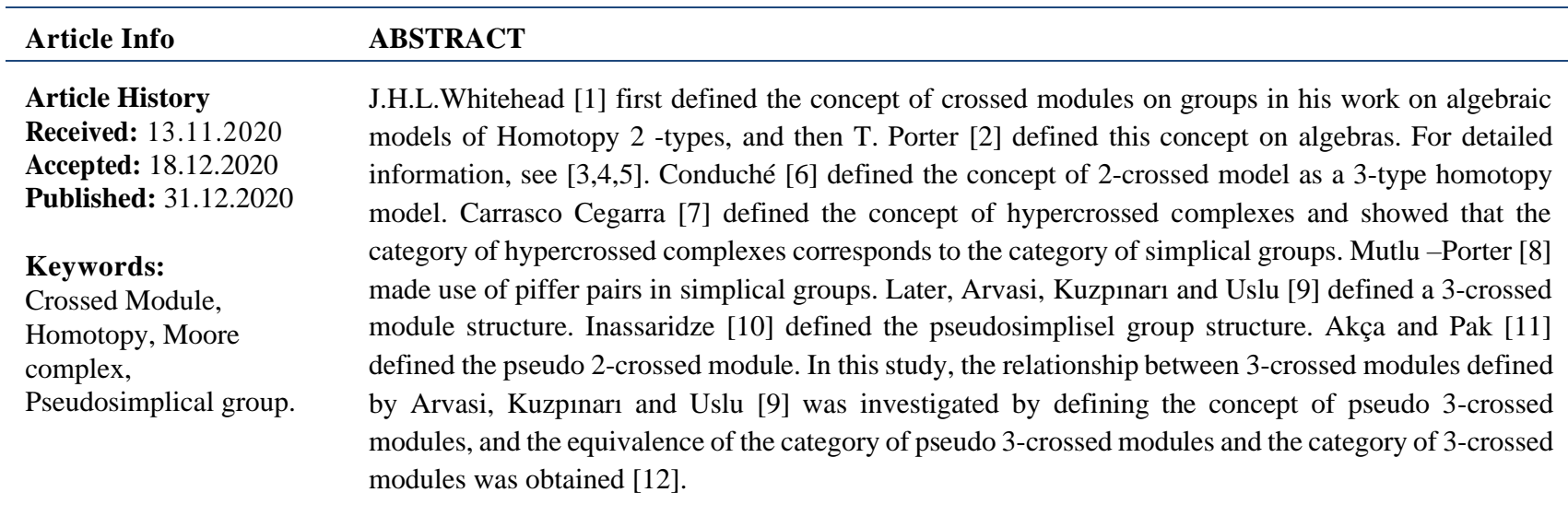

Atıf/Citation: Pak, S.; Gürmen Alansal, Ö.; Cesur, U. (2020). Pseudo 2- Çaprazlanmış Modüller ve Pseudo 3- Çaprazlanmış Modüller, Necmettin Erbakan Üniversitesi Fen ve Mühendislik Bilimleri Dergisi, 2(2), 22-37. 


\section{GIRISŞ (INTRODUCTION)}

\subsection{Pseudosimplisel Gruplar (Pseudosimplicial Groups)}

Boştan farklı bir $G$ grubu üzerinde tanımlanan yüz homomorfizmleri $\partial_{i}^{n}: G_{n} \rightarrow G_{n-1}, 0 \leq i \leq n, n \neq 0$ ve $s_{i}^{n}: G_{n} \rightarrow G_{n+1}, 0 \leq i \leq n$ pseudo dejenere operatörleri ile birlikte $\left\{G_{n}\right\}$ 'den oluşan pseudosimlisel grup yapısı, aşağıdaki özellikleri sağlar:

$$
\begin{aligned}
& \partial_{i}^{n-1} \partial_{j}^{n}=\partial_{j-1}^{n-1} \partial_{i}^{n}, \quad i<j \\
& \partial_{i}^{n+1} s_{j}^{n}=s_{j-1}^{n-1} \partial_{i}^{n}, \quad i<j \\
& \partial_{i}^{n+1} s_{j}^{n}=1=\partial_{i+1}^{n+1} s_{j}^{n} \\
& \partial_{i}^{n+1} s_{j}^{n}=s_{j}^{n-1} \partial_{i-1}^{n}, \quad i>j+1
\end{aligned}
$$

$\mathrm{Bu}$ şartlara,

$$
s_{i}^{n+1} s_{j}^{n}=s_{j+1}^{n+1} s_{i}^{n} \quad i \leq j
$$

şartını da dahil edersek, simplisel grupları elde ederiz. Doğal olarak, her simplisel grup bir pseudosimplisel gruptur. Bunun tersi daima doğru değildir [10].

\subsection{Moore Kompleks (The Moore Complex)}

Bir G pseudosimplisel grubu için,

$$
\left(N G_{n}\right)=\bigcap_{i=0}^{n-1} \operatorname{Kerd}_{i}^{n}
$$

olmak üzere,

$$
(N G, \partial): \cdots \longrightarrow N G_{1} \stackrel{d_{2}}{\longrightarrow} N G_{1} \stackrel{d_{1}}{\longrightarrow} N G_{0}
$$

biçimindeki zincir kompleksine $\mathbf{G}$ nin Moore Kompleksi denir ve $(\boldsymbol{N G}, \boldsymbol{\partial})$ ile gösterilir. G pseudosimplisel grubunun $\boldsymbol{n}$. Homotopi modülü $\boldsymbol{\pi}_{\boldsymbol{n}}(\boldsymbol{G})$,

$$
\begin{aligned}
\pi_{n}(G) \cong H_{n}(N G, \partial) & =\frac{\bigcap_{i=0}^{n} \operatorname{Kerd}_{i}^{n}}{d_{n+1}^{n+1}\left(\bigcap_{i=0}^{n} \operatorname{Kerd}_{i}^{n+1}\right)} \\
& =\frac{N G_{n} \cap \operatorname{Kerd}_{n}^{n}}{d_{n+1}^{n+1}\left(N G_{n+1}\right)}
\end{aligned}
$$

olarak tanımlanır ve bu G nin Moore kompleksinin $n$. homolojisine denktir. $n>k$ için $G_{n}$ pseudosimplisel grubu sifir grubu ise bu gruba $k$-truncated pseudosimplisel grup denir. Bir $k$-truncated pseudosimplisel grup $\operatorname{tr}_{k} G$ ile gösterilir ve truncation funktoru,

$$
\operatorname{tr}_{k}: \text { PseudosimpGrp } \rightarrow \operatorname{Tr}_{k} \text { PseudosimpGrp }
$$

dır. Bu funktorun sağ adjoint funktoru ile $k$ - coskeleletonu,

$$
\text { Cosk }_{k}: \operatorname{Tr}_{k} \text { PseudosimpGrp } \rightarrow \text { PseudosimpGrp }
$$


ve sol adjoint funktoru ile $k$ - skeletonu,

$$
s k_{k}: \operatorname{Tr}_{k} \text { PseudosimpGrp } \rightarrow \text { PseudosimpGrp }
$$

vardır.

\subsection{Tanım ve Notasyonlar (Definitions and Notations)}

Bir, $[n]=\{0<1<2<\cdots<n\}$ sıralı kümesi için $\delta_{i}^{n}:[n-1] \rightarrow[n], \quad 0 \leq i \leq n$ ve $\alpha_{i}^{n}:[n+1] \rightarrow[n], \quad 0 \leq j \leq n$ dönüşümleri,

$$
\delta_{i}^{n}(x)=\left\{\begin{array}{cc}
x & ; \quad x<i \\
x+1 & ; \quad x \geq i
\end{array}\right.
$$

ve

$$
\alpha_{i}^{n}(x)=\left\{\begin{array}{cc}
x & ; x \leq j \\
x-1 & ; \quad x>j
\end{array}\right.
$$

şeklinde tanımlıdır. Ayrıca $0 \leq r \leq n$ için $f:[n] \rightarrow[n-r]$ şeklindeki $f$ oparatörlerinin kümesi $S(n, n-r)$ olsun. Bu kümenin elemanları arasındaki bileşke işleminden faydalanarak, pseudosimplisel özdeşlikler elde edilir.

$$
j<i \text { için } \alpha_{j} \alpha_{i}=\alpha_{i-1} \alpha_{j} \text { dir. Burada }, \alpha_{j}, a_{i} \text { lerin bileşkesi } f \text { yi verir. }
$$

$$
f=\alpha_{i_{1}} o \alpha_{i_{2}} o \cdots o \alpha_{i_{r}}
$$

olup $f \in S(n, n-r)$ dir. Böylece $S(n, n-r)$ nin bir elemanı,

$$
\left\{\mathrm{i}_{1}, \cdots, \mathrm{i}_{\mathrm{r}}\right\} ; 0 \leq \mathrm{i}_{1}<\mathrm{i}_{2}<\cdots<\mathrm{i}_{\mathrm{r}} \leq \mathrm{n}-1
$$

ile belirlenir. Burada $[n]$ nin elemanları $0 \leq i_{1}<i_{2}<\cdots<i_{r} \leq n-1$ formundaki elemanlardır ve $\left\{i_{1}, \cdots, i_{r}\right\}=\{i: f(i)=f(i+1)\}$ dir. Dolayısıyla; $S(n, n)$ nin tek elemanı özdeşlik dönüşümüdür ve ( ) ile veya $\emptyset_{n}$ ile gösterilir. $S(n, 0)$ in da tek elemanı $(n-1, n-2, \cdots, 0)$ dır. $n \geq 0$ için

$$
S(n)=\bigcup_{0 \leq r \leq n} S(n, n-r)
$$

şeklinde tanımlansın. $\alpha, \beta \in S(n)$ ve $\alpha=\left\{i_{r}, \cdots, i_{1}\right\}$ ve $\beta=\left\{j_{s}, \cdots, j_{1}\right\}$ olsun. $\alpha<\beta$ için, $i_{1}=j_{1}, i_{2}=$ $j_{2}, \cdots, i_{k}=j_{k}$, iken $i_{k+1}=j_{k+1} \cdot r<s$ için $i_{1}=j_{1}, i_{2}=j_{2}, \cdots, i_{r}=j_{r}$ olmalıdır. Bu şekilde bir siralama $S(n)$ kümesini bir sıralı küme yapar. Örneğin

$$
\begin{aligned}
S(2)= & \left\{\emptyset_{2}<(1)<(0)<(1,0)\right\} \\
S(3)= & \left\{\emptyset_{3}<(2)<(1)<(2,1)<(0)<(2,0)<(1,0)<(2,1,0)\right\} \\
S(4)= & \left\{\emptyset_{4}<(3)<(2)<(3,2)<(1)<(3,1)<(2,1)<(3,2,1)<(0)<(3,0)<(2,0)<\right. \\
& (3,2,0)<(1,0)<(3,1,0)<(2,1,0)<(3,2,1,0)\}
\end{aligned}
$$

dir. 
1.4. Pseudosimplisel Grubun Yarı Direkt Ayrışması (The Semidirect Decomposition of a Pseudosimplicial Group)

1.4.1. Tanım (Definition) $G$ bir grup, $n \geq 2$ için, $G_{1}, G_{2}, \cdots, G_{n}$ ler $\mathrm{G}$ nin alt grupları olsun. Eğer

i) Her $1 \leq s \leq n$ için $G_{1} \cdot G_{2} \cdot \cdots \cdot G_{s}, G$ nin bir alt grubu,

ii) $G_{1} \cdot G_{2} \cdots \cdot G_{n}=G$,

iii) $1 \leq s<t \leq n$ için $\left(G_{1} \cdot G_{2} \cdot \cdots \cdot G_{s}\right) \cap G_{t}=1$

şartları sağlanıyorsa $G$ ye $G_{1}, G_{2}, \cdots, G_{n}$ lerin $n$-yarıdirekt çarpımı denir. Bu durum

$$
G=G_{1} \rtimes G_{2} \rtimes \cdots \rtimes G_{n}
$$

şeklinde ifade edilir. Bu durumda $g \in G$ elemanı $g_{i} \in G_{i}$ olmak üzere

şeklinde tek bir biçimde yazılıma sahiptir.

$$
g_{1} \cdot g_{2} \cdot \cdots \cdot g_{n}
$$

1.4.2. Tanım (Definition) $G$ bir pseudosimplisel grup olsun. Herhangi $n \geq 0$ için $G_{n}$ grubu, $G_{n} \cong\left(\cdots\left(N G_{n} \rtimes s_{n-1} N G_{n-1}\right) \rtimes \cdots \rtimes s_{n-2} \cdots s_{0} N G_{1}\right) \rtimes\left(\cdots\left(s_{n-2} N G_{n-1} \rtimes s_{n-1} s_{n-2} N G_{n-2}\right) \rtimes \cdots\right.$ $\left.\rtimes s_{n-1} s_{n-2} \cdots s_{0} N G_{0}\right)$

olarak yazılabilir. Bu yazılıma $G_{n}$ nin yarı direkt ayrışımı denir.

1.4.3. Teorem (Theorem) $G$, bir pseudosimplisel grup olmak üzere $G_{n}$ nin yarı direkt ayrışımı;

şeklindedir. Buna göre özel olarak

$$
G_{n} \cong \operatorname{Kerd}_{n}^{n} \rtimes s_{n-1}^{n-1}\left(G_{n-1}\right)
$$

$$
\begin{aligned}
G_{1} & \cong N G_{1} \rtimes s_{0} N G_{0} \\
G_{2} & \cong\left(N G_{2} \rtimes s_{1} N G_{1}\right) \rtimes\left(s_{0} N G_{1} \rtimes s_{1} s_{0} N G_{0}\right) \\
G_{3} & \cong\left(N G_{3} \rtimes s_{2} N G_{2}\right) \rtimes\left(s_{1} N G_{2} \rtimes s_{2} s_{1} N G_{1}\right) \rtimes\left(\left(s_{0} N G_{2} \rtimes s_{2} s_{0} N G_{1}\right) \rtimes\left(s_{1} s_{0} N G_{1} \rtimes s_{2} s_{1} s_{0} N G_{0}\right)\right) . \\
G_{4} & \cong\left(( ( N G _ { 4 } \rtimes s _ { 3 } N G _ { 3 } ) \rtimes ( s _ { 2 } N G _ { 3 } \rtimes s _ { 3 } s _ { 2 } N G _ { 2 } ) ) \rtimes \left(( s _ { 1 } N G _ { 3 } \rtimes s _ { 3 } s _ { 1 } N G _ { 2 } ) \rtimes \left(s_{2} s_{1} N G_{2}\right.\right.\right. \\
& \left.\left.\left.\rtimes s_{3} s_{2} s_{1} N G_{1}\right)\right)\right) \rtimes s_{0}\left(G_{3} \text { in bir ayrlşıml }\right)
\end{aligned}
$$

olur.

$\alpha=\left(i_{l}, \cdots i_{1}\right) \in S(n)$ için karşılık gelen terim,

$$
s_{\alpha}\left(N G_{n-\# \alpha}\right)=s_{i_{l} \cdots i_{1}}\left(N G_{n-\# \alpha}\right)=s_{i_{l} \cdots i_{1}}\left(N G_{n-\# \alpha}\right)
$$

dir. Ayrıca burada $\# \alpha=l$ dir.

Böylece herhangi bir $x \in G_{n}, y \in N G_{n}$ ve $x_{\alpha} \in N G_{n-\# \alpha}$ için

$$
x=y \prod_{\alpha \in S(n)} s_{\alpha}\left(x_{\alpha}\right)
$$

şeklinde ifade edilir. 


\subsection{Hiperçaprazlanmış Kompleks Çiftleri (Hypercrossed Complex Pairings)}

Hiperçaprazlanmış kompleks çiftleri ile ilgili temel fikir Carrasco ve Cegarra [7,13 ] çalışmalarında karşımıza çıkmıştır. Mutlu ve Porter [8] çalışmalarında Conduché [6], çalışmalarından faydalanarak, hiperçaprazlanmış kompleks çiftlerini oluşturmuşlardır. Hiper çaprazlanmış kompleks çiftleri ile ilgili ayrıntılı bilgi için Mutlu ve Porter [8] ve Carrasco ve Cegarra [7,13] çalışmalarına bakılabilir. $\alpha, \beta \in S(n)$ ve $\alpha=\left(i_{r}, \cdots, i_{1}\right), \beta=\left(j_{s}, \cdots, j_{1}\right)$ için $\alpha \cap \beta=\varnothing$ olmak üzere, $(\alpha, \beta)$ şeklindeki ikililerin kümesini $P(n)$ ile gösterelim. Ayrıca $\# \alpha, \# \beta$ sirasıyla $\alpha$ ve $\beta$ nın eleman sayılarını göstersin.

$$
F_{\alpha, \beta}=N G_{n-\# \alpha} \times N G_{n-\# \beta} \rightarrow N G_{n}
$$

dönüşümü, aşağıdaki değişmeli diyagramdan elde edilir.

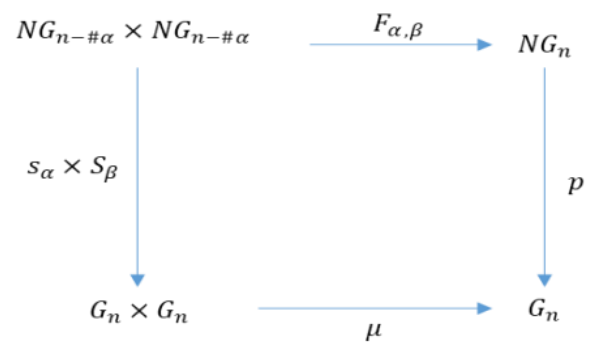

Burada $\quad F_{\alpha, \beta}=p \mu s_{\alpha} \times s_{\beta} \quad$ formunda olup:

$$
\begin{aligned}
& s_{\alpha}=s_{i_{r}} \cdots s_{i_{1}}: N G_{n-\# \alpha} \rightarrow G_{n} \\
& s_{\beta}=s_{j_{s}}, \cdots s_{j_{1}}: N G_{n-\# \beta} \rightarrow G_{n}
\end{aligned}
$$

biçiminde gösterilir.

$$
\begin{aligned}
\mu: G_{n} \times G_{n} & \rightarrow G_{n} \\
x \times y & \mapsto x \cdot y
\end{aligned}
$$

iken $p: G_{n} \rightarrow N G_{n}$ dönüşümü $p=p_{n-1} p_{n-2} \cdots p_{0}$ ve $0 \leq j \leq n-1$ için $p_{j}(x)=x s_{j} d_{j}(x)^{-1} \quad$ şeklinde tanımlıdır.

$x_{\alpha} \in G_{n-\# \alpha}, \quad y_{\beta} \in G_{n-\# \beta}$ olmak üzere; $N G_{n}$ içinde

$$
\begin{aligned}
F_{\alpha, \beta}\left(x_{\alpha}, y_{\beta}\right) & =p \mu\left(s_{\alpha} \times s_{\beta}\right)\left(x_{\alpha}, y_{\beta}\right) \\
& =p\left[s_{\alpha} x_{\alpha}, s_{\beta} y_{\beta}\right]
\end{aligned}
$$

dır. Buradan da $F_{\alpha, \beta}\left(x_{\alpha}, y_{\beta}\right)$ nın elemanları tarafından üretilen $G_{n}$ nin $N_{n}$ normal alt grubunu gösterebiliriz. Bu normal alt grup, $\mathrm{n}=3$ ve $\mathrm{n}=4$ değerleri için şu şekilde elde edilebilir.

$n=3$ için mümkün Peiffer eşleşmeleri,

$$
F_{(1,0)(2)}, F_{(2,0)(1)}, F_{(0)(2,1)}, F_{(0)(2)}, F_{(1)(2)}, F_{(0)(1)} .
$$

dir. $N_{3}$ üreteçleri, her $x_{1} \in N G_{1}, y_{2} \in N G_{2}$ için,

$$
\begin{aligned}
& F_{(1,0)(2)}\left(x_{1}, y_{2}\right)=\left[s_{1} s_{0} x_{1}, s_{2} y_{2}\right]\left[s_{2} y_{2}, s_{2} s_{0} x_{1}\right], \\
& \quad F_{(2,0)(1)}\left(x_{1}, y_{2}\right)=\left[s_{2} s_{0} x_{1}, s_{1} y_{2}\right]\left[s_{1} y_{2}, s_{1} s_{2} x_{1}\right]\left[s_{2} s_{1} x_{1}, s_{2} y_{2}\right]\left[s_{2} y_{2}, s_{2} s_{0} x_{1}\right],
\end{aligned}
$$

her $x_{2} \in N G_{2}, y_{1} \in N G_{1}$ için,

$$
F_{(0)(2,1)}\left(x_{2}, y_{1}\right)=\left[s_{0} x_{2}, s_{2} s_{1} y_{1}\right]\left[s_{2} s_{1} y_{1}, s_{1} x_{2}\right]\left[s_{2} x_{2}, s_{2} s_{1} y_{1}\right],
$$

Ve her $x_{2}, y_{2} \in N G_{2}$ için, 


$$
\begin{aligned}
& F_{(0)(1)}\left(x_{2}, y_{2}\right)=\left[s_{0} x_{2}, s_{1} y_{2}\right]\left[s_{1} y_{2}, s_{1} x_{2}\right]\left[s_{2} x_{2}, s_{2} y_{2}\right], \\
& F_{(0)(2)}\left(x_{2}, y_{2}\right)=\left[s_{0} x_{2}, s_{2} y_{2}\right], \\
& F_{(1)(2)}\left(x_{2}, y_{2}\right)=\left[s_{1} x_{2}, s_{2} y_{2}\right]\left[s_{2} y_{2}, s_{2} x_{2}\right] .
\end{aligned}
$$

şeklindedir.

$n=4$ için, ilgili eşlemeler:

$\begin{array}{lllll}F_{(0)(3,2,1)}, & F_{(3,2,0)(1)}, & F_{(3,1,0)(2),} & F_{(2,1,0)(3),} & F_{(3,0)(2,1)}, \\ F_{(2,0)(3,1)} & F_{(1,0)(3,2)} & F_{(1)(3,2)}, & F_{(0)(3,2)}, & F_{(0)(3,1)}, \\ F_{(0)(2,1)}, & F_{(3,1)(2)}, & F_{(2,1)(3)} & F_{(3,0)(2)}, & F_{(3,0)(1)}, \\ F_{(2,0)(3)}, & F_{(2,0)(1)}, & F_{(1,0)(3),} & F_{(1,0)(2)}, & F_{(2)(3),} \\ F_{(1)(3)}, & F_{(0)(3),} & F_{(1)(2),} & F_{(0)(2)}, & F_{(0)(1)}\end{array}$

şeklindedir. Her $x_{1}, y_{1} \in N G_{1}, x_{2}, y_{2} \in N G_{2}$, ve her $x_{3}, y_{3} \in N G_{3}$, için $N_{4}$ normal alt grubunun üreteçleri kolaylikla elde edilir.

\section{MATERYAL VE YÖNTEM (MATERIALS AND METHODS)}

\section{Groups)}

Pseudosimplisel Gruplar için Çaprazlanmış Modüller (Crossed Modules for Pseudo-simplical

Teorem 2.1. Moore kompleksinin boyu 1 olan Pseudosimplisel gruplar kategorisi ile çaprazlanmış modüller kategorisi denktir [11].

İspat. Kabul edelim ki $G$, Moore kompleksinin boyu 1 olan bir pseudosimplisel grup olsun. $P=$ $N G_{0}=G_{0}, M=N G_{1}=\operatorname{çek}\left(d_{0}: G_{1} \rightarrow G_{0}\right)$ ve $\partial=d_{1}$ alınsın ve $p \in P$ nin $m \in M$ üzerine etkisi

$$
{ }^{p_{m}}=s_{0}(p) m s_{0}(p)^{-1},
$$

olarak alınsın, buna göre

$$
\partial\left({ }^{p} m\right)=d_{1}\left(s_{0}(p) m s_{0}(p)^{-1}\right)
$$

elde edilir. Burada

$$
\ldots \rightarrow 1 \rightarrow M \stackrel{\partial}{\rightarrow} P \rightarrow 1
$$

Moore kompleksinin boyu 1 olduğu için $\partial_{2} N G_{2}=1$ eşitliği mevcuttur. Her $m, m^{\prime} \in M, p \in P$ için

CM1

$$
\begin{aligned}
\partial_{1}\left({ }^{p} m\right) & =d_{1}\left({ }^{p} m\right) \\
= & d_{1}\left(s_{0}(p) m s_{0}(p)^{-1}\right) \\
= & d_{1} s_{0}(p) d_{1}(m) d_{1} s_{0}(p)^{-1} \\
= & p \partial_{1}(m) p^{-1}
\end{aligned}
$$

CM2

$$
\begin{aligned}
{ }^{\left(\partial_{1} m\right)} m^{\prime} & =s_{0} \partial_{1}(m) m^{\prime} s_{0} \partial_{1}(m)^{-1} \\
= & s_{0} d_{1}(m) m^{\prime} s_{0} d_{1}(m)^{-1} \\
= & s_{0} d_{1}(m) m^{\prime} s_{0} d_{1}(m)^{-1}\left[\left(m\left(m^{\prime}\right)^{-1} m^{-1}\right)\left(m m^{\prime} m^{-1}\right)\right]
\end{aligned}
$$




$$
\begin{aligned}
& =d_{2} s_{0}(m) d_{2} s_{1}\left(m^{\prime}\right) d_{2} s_{0}(m)^{-1} d_{2} s_{1}(m) \\
& \qquad d_{2} s_{1}\left(m^{\prime}\right)^{-1} d_{2} s_{1}\left(m^{-1}\right)\left(m m^{\prime} m^{-1}\right) \\
& =d_{2}\left(s_{0}(m) s_{1}\left(m^{\prime}\right) s_{0}(m)^{-1} s_{1}(m) s_{1}\left(m^{\prime}\right)^{-1} s_{1}(m)^{-1}\left(m m^{\prime} m^{-1}\right)\right) \\
& =m m^{\prime} m^{-1}
\end{aligned}
$$

dir. Böylece $\partial: M \rightarrow P$ çaprazlanmış modülü elde edilir.

İlk olarak Conduche [6], homotopi 3 - tipler için bir model olarak 2 - çaprazlanmış modüllerden bahsetmiştir. Akça ve Pak [11] pseudo 2 - çaprazlanmış modülleri tanımlamışlardır. Bu tanımda bir pseudo 2 çaprazlanmış modülün

$$
L \stackrel{\partial_{2}}{\longrightarrow} M \stackrel{\partial_{1}}{\longrightarrow} P
$$

şeklinde grupların bir kompleksi ve $P$-gruplarının $\partial_{2}, \partial_{1}$ morfizmlerinden meydana geldiği ifade edilmiştir. Burada $P$ grubunun $M, L$ ve kendisi üzerine grup etkisi ile birlikte

$$
L \stackrel{\partial_{2}}{\longrightarrow} M
$$

bir pseudo çaprazlanmış modüldür. $M$ nin $L$ üzerinde etkisi ile her $l \in L, m \in M$ ve $p \in P$ için

$$
{ }^{p} m\left({ }^{p} l\right)={ }^{p}\left({ }^{m} l\right)
$$

elde edilir. Ayrıca "Peiffer lifting" dönüşümleri,

$$
\{,\}: M \times M \rightarrow L
$$

her $l, l^{\prime} \in L, m, m^{\prime}, m^{\prime \prime} \in M$ ve $p \in P$ için,

$$
\begin{aligned}
& \text { P-2CM1) } \quad \partial_{2}\left\{m, m^{\prime}\right\}=\left({ }^{\partial_{1} m} m^{\prime}\right) m m^{\prime-1} m^{-1} \\
& \text { P-2CM2) } \quad\left\{\partial_{2} l, \partial_{2} l^{\prime}\right\}=\left[l^{\prime}, l\right] \\
& \text { P-2CM3) (i) } \quad\left\{m m^{\prime}, m^{\prime \prime}\right\}={ }^{\partial_{1} m}\left\{m^{\prime}, m^{\prime \prime}\right\}\left\{m, m^{\prime} m^{\prime \prime} m^{\prime-1}\right\} \\
& \text { (ii) } \quad\left\{m, m^{\prime} m^{\prime \prime}\right\}=\left\{m, m^{\prime}\right\}^{m m^{\prime} m^{-1}}\left\{m, m^{\prime \prime}\right\} \\
& \text { P-2CM4) (a) } \quad\left\{\partial_{2} l, m\right\}={ }^{m} l(l)^{-1} \text {, } \\
& \text { (b) } \quad\left\{m, \partial_{2} l\right\}=\left({ }^{\partial_{1} m} l\right)\left(l^{m}(l)^{-1}\right) \text {. } \\
& \text { P-2CM5) } \quad\left\{m, \partial_{2} l\right\}\left\{\partial_{2} l, m\right\}=\left({ }^{\partial_{1} m} l\right)(l)^{-1}
\end{aligned}
$$

özellikleri sağlanır.

Bir pseudo 2-çaprazlanmış modülü $\left\{L, M, P, \partial_{2}, \partial_{1}\right\}$ şeklinde gösterilebilir. 2 - çaprazlanmış modül tanımını elde etmek için yukarıdaki koşullara

$$
\text { 2CM6) }{ }^{p}\left\{m, m^{\prime}\right\}=\left\{{ }^{p} m,{ }^{p} m^{\prime}\right\}
$$

şartı da eklenmelidir. 
Conduche [6], Moore kompleksinin boyu 2 olan simplisel gruplar kategorisinin 2-çaprazlanmış modüller kategorisine denkliğini göstermiş, Akça ve Pak [11] ise Moore kompleksinin boyu 2 olan pseudo simplisel gruplar kategorisinin pseudo 2- çaprazlanmış modüller kategorisine denkliğini göstermiştir.

Teorem 2.2.1. Pseudo 2-çaprazlanmış modül kategorisi, Moore kompleksinin boyu 2 olan pseudosimplisel gruplar kategorisine denktir [11].

İspat Akça ve Pak [11] tarafından verilmiştir.

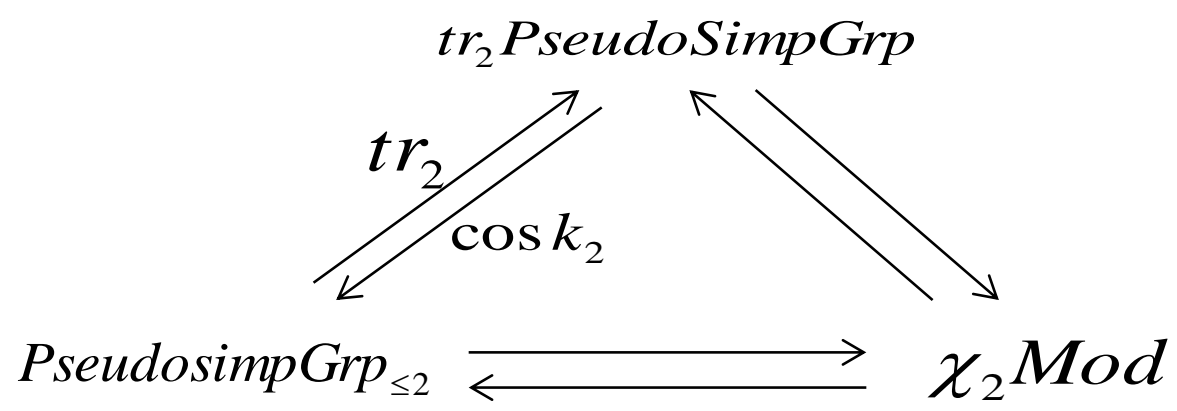

elde edilmiştir.

\section{TARTIŞMA VE SONUÇLAR (DISCUSSION AND CONCLUSIONS)}

\subsection{Pseudo 3-Çaprazlanmış Modüller (Pseudo 3- Crossed Modules)}

Pseudo 3-çaprazlanmış modüller kategorisini oluşturmak için ilk olarak, Conduch'e [6] nin metodundan faydalanacağız. Conduch'e [6] metodunda bir simplisel gurubun yarı direkt ayrışımını kullanarak bazı eşitlikler verdi. Bu eşitliklerin Mutlu ve Porter [8] tarafından $n=3$ için $\partial_{3}$ altında $F_{\alpha, \beta}$ Peiffer çiftlerinin görüntüleri olduğu ifade edilmiştir. Arvasi, Kuzpınarı ve Uslu [9] çalışmalarında Conduch'e [6] den farklı olarak direkt ayrışım yerine $F_{\alpha, \beta}$ kullanmışlardır. Böylece $n=4$ için benzer eşitlikleri tanımlayarak bir 3-çaprazlanmış modülün aksiyomlarını elde etmişlerdir. Bizde Arvasi, Kuzpınarı ve Uslu [9] nun çalışmalarından faydalanarak, Pseudo 3- çaprazlanmış modül aksiyomlarını elde etmeye çalışacağız.

$G$, Moore kompleksinin uzunluğu 3 olan bir pseudosimplisel grup olsun, $N G_{0}=N, N G_{1}=A, N G_{2}=$ $B, N G_{3}=C$ ile bir

$$
C \stackrel{\partial_{3}}{\longrightarrow} B \stackrel{\partial_{2}}{\longrightarrow} A \stackrel{\partial_{1}}{\longrightarrow} N
$$

grup kompleksi elde edilir. Burada $N \operatorname{nin} A, B, C$ üzerine etkisi, $A$ nın $B v e C$ üzerine ve $B$ nin $C$ üzerine etkisi

$$
\begin{aligned}
& n_{a}=s_{0} n(a) s_{0} n^{-1} \\
& n_{b} b=s_{1} s_{0} n(b) s_{1} s_{0} n^{-1} \\
& { }^{n_{c}}=s_{2} s_{1} s_{0} n(c) s_{2} s_{1} s_{0} n^{-1} \\
& \quad a_{b}=s_{1} a(b) s_{1} a^{-1} \\
& a_{c}=s_{2} s_{1} a(c) s_{2} s_{1} a^{-1} \\
& b . c=s_{2} b(c) s_{2} b^{-1}
\end{aligned}
$$

olarak tanımlanır. 


$$
\begin{aligned}
& {\left[s_{1} s_{0} a s_{2} s_{1} \partial_{1} a, c\right]=1} \\
& {\left[s_{1} b s_{2} s_{1} \partial_{2} b, c\right]=1} \\
& {\left[c^{\prime}, c^{-1} s_{2} \partial_{3} c\right]=1}
\end{aligned}
$$

ve buradan

$$
\begin{gathered}
\partial_{1} a{ }_{c}=s_{1} s_{0} a(c) s_{1} s_{0} a^{-1} \\
\partial_{2} b c=s_{1} b(c) s_{1} b^{-1} \\
\partial_{3} c \cdot c^{\prime}=c\left(c^{\prime}\right) c^{-1}
\end{gathered}
$$

dir ve

$$
\begin{aligned}
\partial_{3}(b . c) & =\partial_{3}\left(s_{2} b(c) s_{2} b^{-1}\right) \\
& =\partial_{3} s_{2} b\left(\partial_{3} c\right) s_{2} b^{-1} \\
& =b\left(\partial_{3} c\right) b^{-1}
\end{aligned}
$$

elde edilir. Böylece $\partial_{3}: C \rightarrow B$ çaprazlanmış modül olduğu gösterilmiş olur.

\subsection{Tanım}

$$
C \stackrel{\partial_{3}}{\longrightarrow} B \stackrel{\partial_{2}}{\longrightarrow} A \stackrel{\partial_{1}}{\longrightarrow} N
$$

yukarıdaki gibi bir grup kompleksi olmak üzere, Peiffer liftingleri $a, a^{\prime} \in A, v e b, b^{\prime} \in B$ için

$$
\begin{aligned}
& \{,\} \quad: A \times A \rightarrow B \\
& \left\{a, a^{\prime}\right\}=\left[s_{1} a, s_{1} a^{\prime}\right]\left[s_{1} a^{\prime}, s_{0} a\right] \\
& \{,\}_{(1)(0)} \quad: B \times B \rightarrow C \\
& \left\{b, b^{\prime}\right\}_{(1)(0)}=\left[s_{2} b^{\prime}, s_{2} b\right]\left[s_{1} b, s_{1} b^{\prime}\right]\left[s_{1} b^{\prime}, s_{0} b\right] \\
& \{,\}_{(2)(1)} \quad: B \times B \rightarrow C \\
& \left\{b, b^{\prime}\right\}_{(2)(1)}=\left[s_{2} b, s_{2} b^{\prime}\right]\left[s_{2} b^{\prime}, s_{1} b\right] \\
& \{,\}_{(0)(2)} \quad: B \times B \rightarrow C \\
& \left\{b, b^{\prime}\right\}_{(0)(2)}=\left[s_{2} b^{\prime}, s_{0} b\right] \\
& \{,\}_{(1,0)(2)}: A \times B \rightarrow C \\
& \left\{a, b^{\prime}\right\}_{(1,0)(2)}=\left[s_{2} s_{0} a, s_{2} b^{\prime}\right]\left[s_{2} b^{\prime}, s_{1} s_{0} a\right] \\
& \{,\}_{(2,0)(1)}: A \times B \rightarrow C \\
& \left\{a, b^{\prime}\right\}_{(2,0)(1)}=\left[s_{2} s_{0} a, s_{2} b^{\prime}\right]\left[s_{2} b^{\prime}, s_{2} s_{1} a\right]\left[s_{2} s_{1} a, s_{1} b^{\prime}\right]\left[s_{1} b^{\prime}, s_{2} s_{0} a\right] \\
& \{,\}_{(2,0)(1)}: B \times A \rightarrow C \\
& \left\{b^{\prime}, a\right\}_{(0)(2,1)}=\left[s_{2} s_{1} a, s_{2} b^{\prime}\right]\left[s_{1} b^{\prime}, s_{2} s_{1} a\right]\left[s_{2} s_{1} a, s_{0} b^{\prime}\right]
\end{aligned}
$$

şeklinde tanımlansın. Böylece aşağıdaki özdeşlikler elde edilir. Burada, $a, a^{\prime}, a^{\prime \prime} \in A, b, b^{\prime}, b^{\prime \prime} \in B$, $c, c^{\prime}, c^{\prime \prime} \in C$ olmak üzere 


\begin{tabular}{|c|c|c|}
\hline$\left\{\boldsymbol{a}, \boldsymbol{\partial}_{3} c^{\prime \prime}\right\}_{(\mathbf{1}, \mathbf{0})(2)}$ & $=$ & $\left\{\boldsymbol{a}, \boldsymbol{\partial}_{3} c^{\prime \prime}\right\}_{(2,0)(1)} a_{\left(c^{\prime \prime}\right)^{\boldsymbol{\partial}_{1} \boldsymbol{a}}\left(c^{\prime \prime}-\mathbf{1}\right)}$ \\
\hline$\left\{\boldsymbol{\partial}_{3} c^{\prime \prime}, \boldsymbol{a}\right\}_{(\mathbf{0})(2, \mathbf{1})}$ & $=$ & ${ }^{a}\left(c^{\prime}\right) c^{\prime-1}$ \\
\hline$\left\{a, \partial_{3} c\right\}_{(1,0)(2)}$ & $=$ & $\left\{a, \partial_{3} c\right\}_{(2,0)(1)}\left\{\partial_{3} c, a\right\}_{(0)(2,1)} \quad c^{\partial_{1} m} c^{-1}$ \\
\hline$\left\{b^{\prime}, \boldsymbol{\partial}_{2} \boldsymbol{b}\right\}_{(\mathbf{0})(2,1)}$ & $=$ & $\left\{b, \boldsymbol{b}^{\prime}\right\}_{(2)(1)}^{-1}\left\{\boldsymbol{b}^{\prime}, \boldsymbol{b}\right\}_{(\mathbf{1})(\mathbf{0})}$ \\
\hline$\left\{\boldsymbol{\partial}_{2} \boldsymbol{b}, \boldsymbol{b}^{\prime}\right\}_{(2,0)(1)}$ & $=$ & $\left\{\boldsymbol{b}, \boldsymbol{b}^{\prime}\right\}_{(\mathbf{0})(2)}^{-1}{ }^{\left[\boldsymbol{b}^{\prime}, b\right]}\left(\left\{b, \boldsymbol{b}^{\prime}\right\}_{(2)(\mathbf{1})}\right) \quad\left\{\boldsymbol{b}, \boldsymbol{b}^{\prime}\right\}_{(\mathbf{1})(\mathbf{0})}$ \\
\hline$\left\{\boldsymbol{\partial}_{2} \boldsymbol{b}, \boldsymbol{b}^{\prime}\right\}_{(1,0)(2)}$ & $=$ & $\left(\left\{\boldsymbol{b}, \boldsymbol{b}^{\prime}\right\}_{(0)(2)}\right)^{-1}$ \\
\hline$\left\{b, \boldsymbol{b}^{\prime}, \boldsymbol{b}^{\prime \prime}\right\}_{(2)(\mathbf{1})}$ & $=$ & $\left\{\boldsymbol{b}, \boldsymbol{b}^{\prime}\right\}_{(2)(1)}{ }^{\partial \boldsymbol{b}} \boldsymbol{b}^{\prime} .\left\{b, \boldsymbol{b}^{\prime \prime}\right\}_{(2)(1)}$ \\
\hline$\left\{\boldsymbol{b} \boldsymbol{b}^{\prime}, \boldsymbol{b}^{\prime \prime}\right\}_{(2)(1)}$ & $=$ & b. $\left\{\boldsymbol{b}^{\prime}, \boldsymbol{b}^{\prime \prime}\right\}_{(2)(1)}\left\{\boldsymbol{b}^{\partial \boldsymbol{b}^{\prime}} \boldsymbol{b}^{\prime \prime}\right\}_{(2)(1)}$ \\
\hline $\boldsymbol{\partial}_{3}\left(\left\{\boldsymbol{b}, \boldsymbol{b}^{\prime}\right\}_{(\mathbf{1})(\mathbf{0})}\right)$ & $=$ & {$\left[\boldsymbol{b}, \boldsymbol{b}^{\prime}\right]\left\{\boldsymbol{\partial}_{2} \boldsymbol{b}, \boldsymbol{\partial}_{2} \boldsymbol{b}^{\prime}\right.$} \\
\hline$\partial_{3}\left(\left\{\boldsymbol{b}, \boldsymbol{b}^{\prime}\right\}_{(2)(1)}\right)$ & $=$ & $b b^{\prime} b^{-1}\left({ }^{d_{2} b} b^{\prime}\right)^{-1}$ \\
\hline $\boldsymbol{\partial}_{3}\left(\left\{b, \boldsymbol{b}^{\prime}\right\}_{(\mathbf{0})(2)}\right)$ & $=$ & $\boldsymbol{\partial}_{3}\left(\left\{\boldsymbol{\partial}_{2} b, \boldsymbol{b}^{\prime}\right\}_{(\mathbf{1}, \mathbf{0})(\mathbf{2})}\right)^{-\mathbf{1}}$ \\
\hline $\boldsymbol{\partial}_{3}\left(\{b, a\}_{(\mathbf{0})(2,1)}\right)$ & $=$ & ${ }^{\boldsymbol{a}} \boldsymbol{b} \boldsymbol{b}^{-1}\left\{\boldsymbol{\partial}_{2} b, a\right\}$ \\
\hline $\boldsymbol{\partial}_{3}\left(\{a, b\}_{(2,0)(1)}\right)$ & $=$ & $\partial_{3}\{a, b\}_{(1,0)(2)}{ }^{\partial_{1} a} b^{a}\left(b^{-1}\right)\left\{a, \partial_{2} b\right\}$ \\
\hline$\left\{\partial_{3} c, b\right\}_{(2)(1)}\left\{b, \partial_{3} c\right\}_{(2)(1)}$ & $=$ & $c\left({ }^{\partial_{2} b}\left(c^{-1}\right)\right)$ \\
\hline$\left\{\boldsymbol{\partial}_{3} \boldsymbol{c}, b\right\}_{(\mathbf{1})(\mathbf{0})}\left\{b, \boldsymbol{\partial}_{3} \boldsymbol{c}\right\}_{(\mathbf{1})(\mathbf{0})}$ & $=$ & 1 \\
\hline$\left\{\partial_{3} c, \partial_{3} c^{\prime}\right\}_{(2)(1)}$ & $=$ & {$\left[c, c^{\prime}\right]$} \\
\hline$\left\{\partial_{3} c, \partial_{3} c^{\prime}\right\}_{(1)(0)}$ & $=$ & {$\left[c^{\prime}, c\right]$} \\
\hline$\left\{\boldsymbol{\partial}_{3} \boldsymbol{c}, \boldsymbol{b}^{\prime}\right\}_{(\mathbf{0})(2)}$ & $=$ & 1 \\
\hline$\left\{\partial_{2} b, \partial_{3} c\right\}_{(1,0)(2)}$ & $=$ & $\left\{b, \partial_{3} c\right\}_{(0)(2)}^{-1}$ \\
\hline$\left\{\partial_{2} b, \partial_{3} c\right\}_{(2,0)(1)}$ & $=$ & $\left\{b, \partial_{3} c\right\}_{(0)(2)} c\left({ }^{\partial_{2} b}\left(c^{-1}\right)\right)$ \\
\hline$\left\{\partial_{3} c, \partial_{2} b\right\}_{(0)(2,1)}$ & $=$ & $\partial_{2} b c c^{-1}$ \\
\hline
\end{tabular}




\begin{tabular}{|c|}
\hline${ }^{n}\left\{a, \boldsymbol{a}^{\prime}\right\}=\left\{{ }^{n} \boldsymbol{a},{ }^{n} \boldsymbol{a}^{\prime}\right\}$ \\
\hline${ }^{\left.n_{\{b}, \boldsymbol{b}^{\prime}\right\}_{(\mathbf{1})(\mathbf{0})}}=\left\{{ }^{\boldsymbol{n}} \boldsymbol{b},{ }^{n} \boldsymbol{b}^{\prime}\right\}_{(\mathbf{1})(\mathbf{0})}$ \\
\hline${ }^{n}\left\{b, b^{\prime}\right\}_{(2)(1)}=\left\{{ }^{n} b^{n} b^{\prime}\right\}_{(2)(1)}$ \\
\hline${ }^{n}\left\{\boldsymbol{b}, \boldsymbol{b}^{\prime}\right\}_{(\mathbf{0})(2)}=\left\{{ }^{n} \boldsymbol{b},{ }^{n} \boldsymbol{b}^{\prime}\right\}_{(0)(2)}$ \\
\hline$n_{\left\{a, b^{\prime}\right\}_{(1,0)(2)}}=\left\{{ }^{n} \boldsymbol{a},{ }^{n} b^{\prime}\right\}_{(1,0)(2)}$ \\
\hline${ }^{n}\left\{a, b^{\prime}\right\}_{(2,0)(1)}=\left\{{ }^{n} a,{ }^{n} b^{\prime}\right\}_{(2,0)(1)}$ \\
\hline$n_{\left\{\boldsymbol{b}^{\prime}, a\right\}_{(\mathbf{0})(2,1)}}=\left\{{ }^{n} \boldsymbol{b}^{\prime},{ }^{n} \boldsymbol{a}\right\}_{(\mathbf{0})(2, \mathbf{1})}$ \\
\hline
\end{tabular}

\begin{tabular}{|c|}
\hline${ }^{n}\left\{\boldsymbol{a}^{\prime}, \boldsymbol{a}^{\prime \prime}\right\}={ }^{\boldsymbol{a}}\left\{\boldsymbol{a}^{\prime}, \boldsymbol{a}^{\boldsymbol{a}} \boldsymbol{a}^{\prime \prime}\right\}$ \\
\hline$\left.{ }^{n_{\{b}}, \boldsymbol{b}^{\prime}\right\}_{(\mathbf{1})(\mathbf{0})}=\left\{{ }^{a} \boldsymbol{b},{ }^{a} \boldsymbol{b}^{\prime}\right\}_{(\mathbf{1})(\mathbf{0})}$ \\
\hline${ }^{n}\left\{b, b^{\prime}\right\}_{(2)(1)}=\left\{{ }^{a} b,{ }^{a} b^{\prime}\right\}_{(2)(1)}$ \\
\hline${ }^{n}\left\{\boldsymbol{b}, \boldsymbol{b}^{\prime}\right\}_{(\mathbf{0})(2)}=\left\{{ }^{a} \boldsymbol{b},{ }^{a} \boldsymbol{b}^{\prime}\right\}_{(\mathbf{0})(2)}$ \\
\hline${ }^{n}\left\{a, b^{\prime}\right\}_{(1,0)(2)}=\left\{{ }^{a} a,{ }^{a} b^{\prime}\right\}_{(1,0)(2)}$ \\
\hline$\left.{ }^{n_{\{a}} \boldsymbol{b}^{\prime}\right\}_{(2,0)(1)}=\left\{{ }^{a} a,{ }^{a} b^{\prime}\right\}_{(2,0)(1)}$ \\
\hline${ }^{\left.n_{\left\{b^{\prime}\right.}, a\right\}_{(\mathbf{0})(2,1)}}=\left\{{ }^{a} \boldsymbol{b}^{\prime},{ }^{a} \boldsymbol{a}\right\}_{(\mathbf{0})(2, \mathbf{1})}$ \\
\hline
\end{tabular}

elde edilir.

\subsection{Tanım Grupların bir kompleksinin 3 - çaprazlanmış modülü}

$$
C \stackrel{\partial_{3}}{\longrightarrow} B \stackrel{\partial_{2}}{\longrightarrow} A \stackrel{\partial_{1}}{\longrightarrow} N
$$

grupların ve morfizmlerinin bir kompleksi olsun. $N \operatorname{nin} C$, B ve $A$ üzerine etkisi, $A \operatorname{nin} C$ ve $B$ üzerine etkisi ve $B \operatorname{nin} C$ üzerine etkisi ile birlikte

$$
\begin{gathered}
\{,\}_{(1)(0)}: B \times B \rightarrow C \\
\{,\}_{(1,0)(2)}: A \times B \rightarrow C \\
\{,\}_{(0)(2,1)}: B \times A \rightarrow C \\
\{,\}_{(0)(2)}: B \times B \rightarrow C \\
\{,\}_{(2)(1)}: B \times B \rightarrow C \\
\{,\}_{(2,0)(1)}: A \times B \rightarrow C \\
\{,\}: A \times A \rightarrow B
\end{gathered}
$$


3 boyutlu Peiffer liftingi denilen dönüşümler için
P-3CM1)

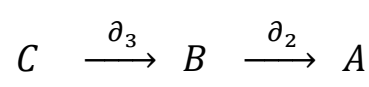

$\{\quad,\}_{(2)(1)}$ Peiffer lifting ile bir pseudo 2 çaprazlanmış modüldür.

$$
\begin{array}{ll}
\text { P-3CM2) } & \left\{a, \partial_{3} c\right\}_{(1,0)(2)}=\left\{a, \partial_{3} c\right\}_{(2,0)(1)}{ }^{a}(c)^{\partial_{1} a}\left(c^{-1}\right) \\
\text { P-3CM3) } & \left\{\partial_{3} c, a\right\}_{(0)(2,1)}=a(c) c^{-1} \\
\text { P-3CM4) } & \left\{a, \partial_{3} c\right\}_{(1,0)(2)}=\left\{a, \partial_{3} c\right\}_{(2,0)(1)}\left\{\partial_{3} c, a\right\}_{(0)(2,1)}(c)^{\partial_{1} a}\left(c^{-1}\right) \\
\text { P-3CM5) } & \left\{b^{\prime}, \partial_{3} b\right\}_{(0)(2,1)}=\left\{b, b^{\prime}\right\}_{(2)(1)}^{-1}\left\{b^{\prime}, b\right\}_{(1)(0)} \\
\text { P-3CM6) } & \multicolumn{1}{c}{\partial_{2} b, b^{\prime}}_{(2,0)(1)}=\left\{b, b^{\prime}\right\}_{(0)(2)}^{-1}{ }^{\left[b^{\prime}, b\right]}\left(\left\{b^{\prime}, b\right\}_{(2)(1)}\right)\left\{b, b^{\prime}\right\}_{(1)(0)} \\
\text { P-3CM7) } & \left\{\partial_{2} b, b^{\prime}\right\}_{(1,0)(2)}=\left(\left\{b, b^{\prime}\right\}_{(0)(2)}\right)^{-1} \\
\text { P-3CM8) } & \partial_{3}\left(\left\{b, b^{\prime}\right\}_{(1),(0)}\right)=\left[b, b^{\prime}\right]\left\{\partial_{2} b, \partial_{2} b^{\prime}\right\} \\
\text { P-3CM9) } & \partial_{3}\left(\left\{b, b^{\prime}\right\}_{(0),(2)}\right)=\partial_{3}\left(\left\{\partial_{2} b, b^{\prime}\right\}_{(1,0)(2)}\right)^{-1} \\
\text { P-3CM10) } & \partial_{3}\left(\{b, a\}_{(0),(2,1)}\right)=a b b^{-1}\left\{\partial_{2} b, a\right\} \\
\text { P-3CM11) } & \partial_{3}\left(\{a, b\}_{(2,0),(1)}\right)=\partial_{3}\{a, b\}_{(1,0)(2)} \partial_{1} a b^{a} b^{-1}\left\{a, \partial_{2} b\right\} \\
\text { P-3CM12a) } & \left\{\partial_{3} c, b\right\}_{(1)(0)}=\left({ }^{b} c\right) c^{-1} \\
\text { P-3CM12b) } & \left\{b, \partial_{3} c\right\}_{(1)(0)}=c\left({ }^{b} c\right)^{-1} \\
\text { P-3CM13) } & \left\{\partial_{3} c, \partial_{3} c^{\prime}\right\}_{(1)(0)}=\left[c^{\prime}, c\right] \\
\text { P-3CM14) } & \left\{\partial_{3} c, b^{\prime}\right\}_{(0)(2)}=1 \\
\text { P-3CM15) } & \left\{\partial_{3} b, \partial_{3} c\right\}_{(1,0)(2)}=\left\{b, \partial_{3}\right\}_{(0)(2)}{ }^{-1} \\
\text { P-3CM16) } & \left\{\partial_{2} b, \partial_{3} c\right\}_{(2,0)(1)}=\left\{b, \partial_{3}\right\}_{(0)(2)} c\left(^{\partial_{2} b}\left(c^{-1}\right)\right) \\
\text { P-3CM17) } & \left\{\partial_{3} c, \partial_{2} b\right\}_{(0)(2,1)}={ }^{2} b c c^{-1} \\
\text { P-3CM18) } & \partial_{2}\left\{a, a^{\prime}\right\}^{\prime}=a a^{\prime} a^{-1}\left({ }^{\partial_{1} a} a^{\prime}\right)^{-1} \\
\text { PCI } &
\end{array}
$$

aksiyomları sağlanıyorsa yukarıdaki komplekse bir pseudo 3-çaprazlanmış modül ve $\left(C, B, A, N, \partial_{3}, \partial_{2}, \partial_{1}\right)$ ile gösterilir.

Gruplar için pseudo 3-çaprazlanmış modül morfizimlerini,

gösterebiliriz.

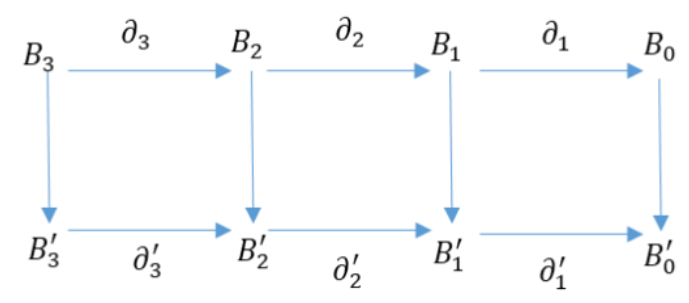




$$
\text { - } f_{1}\left({ }^{n} a\right)={ }^{\left(f_{0}(n)\right)} f_{1}(a), \quad f_{2}\left({ }^{n} b\right)={ }^{\left(f_{0}(n)\right)} f_{2}(b), \quad f_{3}\left({ }^{n} c\right)={ }^{\left(f_{0}(n)\right)} f_{3}(c)
$$

Her $c \in C, b \in B, a \in A$ için,

$$
\begin{aligned}
& \left.\{,\} f_{2} \times f_{2}=f_{3}\{,\} \text { için }\{,\}_{(0)(2),\{,}\right\}_{(2)(1)},\{,\}_{(1)(0)} \\
& \{,\} f_{1} \times f_{2}=f_{3}\{,\} \text { için }\{,\}_{(1,0)(2)},\{,\}_{(2,0)(1)} \\
& \{,\} f_{2} \times f_{1}=f_{3}\{,\} \text { için }\{,\}_{(0)(2,1)} \\
& \{,\} f_{1} \times f_{1}=f_{2}\{,\} \text { için }\{,\}
\end{aligned}
$$

Böylece, $\boldsymbol{p} \boldsymbol{X}_{\mathbf{3}} \boldsymbol{M o d}$ ile göstereceğimiz pseudo 3-çaprazlanmış modüller kategorisini tanımlayabiliriz.

\section{Pseudosimplisel Gruplar (Pseudosimplicial Groups)}

Bir G, Moore kompleksi NG ile gösterilen bir pseudosimplisel grup olsun,

$$
N G_{3} / \partial_{4}\left(N G_{4} \cap D_{4}\right) \stackrel{\partial_{3}}{\longrightarrow} N G_{2} \stackrel{\partial_{2}}{\longrightarrow} N G_{1} \stackrel{\partial_{1}}{\longrightarrow} N G_{0}
$$

grup kompleksi

$$
\begin{aligned}
& \left\{\quad, \quad: N G_{1} \times N G_{1} \rightarrow N G_{2}\right. \\
& \left\{x_{1}, y_{1}\right\}=\left[s_{0} x_{1}, s_{1} y_{1}\right]\left[s_{1} y_{1}, s_{0} x_{1}\right] \\
& \{,\}_{(1)(0)}: N G_{2} \times N G_{2} \rightarrow N G_{3} / \partial_{4}\left(N G_{4} \cap D_{4}\right) \\
& \left\{x_{2}, y_{2}\right\}=\overline{\left[s_{0} x_{2}, s_{1} y_{2}\right]\left[s_{1} y_{2}, s_{1} x_{2}\right]\left[s_{2} x_{2}, s_{2} y_{2}\right]} \\
& \{,\}_{(2)(1)}: N G_{2} \times N G_{2} \rightarrow N G_{3} / \partial_{4}\left(N G_{4} \cap D_{4}\right) \\
& \left\{x_{2}, y_{2}\right\}=\overline{\left[s_{1} x_{2}, s_{2} y_{2}\right]\left[s_{2} y_{2}, s_{2} x_{2}\right]} \\
& \{,\}_{(0)(2)}: N G_{2} \times N G_{2} \rightarrow N G_{3} / \partial_{4}\left(N G_{4} \cap D_{4}\right) \\
& \left\{x_{2}, y_{2}\right\}=\overline{\left[s_{0} x_{2}, s_{2} y_{2}\right]} \\
& \{\quad,\}_{(1,0)(2)}: N G_{1} \times N G_{2} \rightarrow N G_{3} / \partial_{4}\left(N G_{4} \cap D_{4}\right) \\
& \left\{x_{1}, y_{2}\right\}=\overline{\left[s_{1} s_{0} x_{1}, s_{2} y_{2}\right]\left[s_{2} y_{2}, s_{2} s_{0} x_{1}\right]} \\
& \{\quad,\}_{(2,0)(1)}: N G_{1} \times N G_{2} \rightarrow N G_{3} / \partial_{4}\left(N G_{4} \cap D_{4}\right) \\
& \left\{x_{1}, y_{2}\right\}=\overline{\left[s_{2} s_{0} x_{1}, s_{1} y_{2}\right]\left[s_{1} y_{2}, s_{2} s_{1} x_{1}\right]\left[s_{2} s_{1} x_{1}, s_{2} y_{2}\right]\left[s_{2} y_{2}, s_{2} s_{0} x_{1}\right]} \\
& \{\quad,\}_{(0)(2,1)}: N G_{2} \times N G_{1} \rightarrow N G_{3} / \partial_{4}\left(N G_{4} \cap D_{4}\right) \\
& \left\{y_{2}, x_{1}\right\}=\overline{\left[s_{0} y_{2}, s_{2} s_{1} x_{1}\right]\left[s_{2} s_{1} x_{1}, s_{1} y_{2}\right]\left[s_{2} y_{2}, s_{2} s_{1} x_{1}\right]}
\end{aligned}
$$

Peiffer liftingleri ile birlikte bir pseudo 3 - çaprazlanmış modüldür.

3.4. Teorem Pseudo 3 - çaprazlanmış modüller kategorisi, Moore kompleksinin uzunluğu 3 olan pseudosimplisel gruplar kategorisine denktir. 
İspat: G, Moore kompleksinin uzunluğu 3 olan bir pseudosimplisel grup olsun.

$$
N G_{3} \stackrel{\partial_{3}}{\longrightarrow} N G_{2} \stackrel{\partial_{2}}{\longrightarrow} N G_{1} \stackrel{\partial_{1}}{\longrightarrow} N G_{0}
$$

grup kompleksinin bir pseudo 3 - çaprazlanmış modül olduğunu gösterelim. Moore kompleksinin uzunluğu 3 olduğundan, $N G_{4} \cap D_{4}=1$, böylece $\partial_{4}\left(N G_{4} \cap D_{4}\right)=1$ elde edilir ve $N G_{3} / \partial_{4}\left(N G_{4} \cap D_{4}\right)$ yerine $N G_{3}$ alabiliriz $N G_{4} \cap D_{4}$,

$$
7_{3}: \text { PSimpGrp } \leq 3 \rightarrow \text { p } \mathbf{X}_{3} \text { Mod }
$$

Böylece Moore kompleksinin uzunluğu 3 olan pseudsimplisel gruplar kategorisinden pseudo 3 çaprazlanmış modüller kategorisine bir funktor vardır. Tersine;

$$
C \stackrel{\partial_{3}}{\longrightarrow} B \stackrel{\partial_{2}}{\longrightarrow} A \stackrel{\partial_{1}}{\longrightarrow} N
$$

Bir pseudo 3 çaprazlanmış modül olsun. $H_{0}=N$ olmak üzere, $N$ nin $A$ üzerine etkisi ile $H_{1}=A \rtimes N$ yarı direk çarpımı elde edilir

$$
\begin{gathered}
d_{0}: A \rtimes N \rightarrow N \\
(a, n) \mapsto n \\
d_{1}: A \rtimes N \rightarrow N \\
(a, n) \mapsto\left(\partial_{1}(a)\right) n \\
s_{0}: N \rightarrow A \rtimes N \\
n \mapsto(1, n) .
\end{gathered}
$$

$(a, n) \in A \rtimes N$ için yüz ve dejenere dönüşümleri yazılabilir. Şimdi, $A$ ve $N$ 'nin $B$ üzerine etkisi ile, $H_{2}=$ $(B \rtimes A) \times(A \rtimes N)$ yarı-direkt çarpımını elde ederiz. $b \in B, a, a^{\prime} \in A$ ve $n \in N$ için yüz ve dejenere dönüşümleri,

$$
\begin{gathered}
d_{0}:(B \rtimes A) \rtimes(A \rtimes N) \rightarrow(A \rtimes N) \\
\left(b, a, a^{\prime}, n\right) \rightarrow\left(a^{\prime}, n\right) \\
d_{1}:(B \rtimes A) \rtimes(A \rtimes N) \rightarrow(A \rtimes N) \\
\left(b, a, a^{\prime}, n\right) \rightarrow\left(a a^{\prime}, n\right) \\
d_{2}:(B \rtimes A) \rtimes(A \rtimes N) \rightarrow(A \rtimes N) \\
\left(b, a, a^{\prime}, n\right) \rightarrow\left(\partial_{2}(b) a, \partial_{1}\left(a^{\prime}\right) n\right) \\
s_{0}:(A \rtimes N) \rightarrow(B \rtimes A) \rtimes(A \rtimes N) \\
\left(a^{\prime}, n\right) \rightarrow\left(1,1, a^{\prime}, n\right) \\
s_{1}:(A \rtimes N) \rightarrow(B \rtimes A) \rtimes(A \rtimes N) \\
\left(a^{\prime}, n\right) \quad \rightarrow\left(1, a^{\prime}, 1, n\right)
\end{gathered}
$$


$\{,\}_{(2)(1)}$ bir pseudo 2 - çaprazlanmış modül olduğundan $B \operatorname{nin} C$ üzerine etkisi, $b \in B, c \in C$ için

$$
{ }^{b} c=\left\{\partial_{3} c, b\right\}_{(2)(1)} c^{-1}
$$

bu etkiyi kullanarak $C \rtimes B$ yarı direkt çarpımını elde ederiz. $(b, a) \in B \rtimes A$ nın $(c, b) \in C \rtimes B$ üzerine etkisini kullanarak

$$
\begin{aligned}
{ }^{(1, a)}\left(c, b^{\prime}\right) & ={ }^{a}\left({ }^{1} c\right),{ }^{a}\left({ }^{1} b^{\prime}\right) \\
& ={ }^{a}(c),{ }^{a}\left(b^{\prime}\right) \\
{ }^{(b, 1)}\left(c, b^{\prime}\right) & ={ }^{1}\left({ }^{b} c\right),{ }^{1}\left({ }^{b} b^{\prime}\right) \\
& ={ }^{b}(c),{ }^{b}\left(b^{\prime}\right) \\
& ={ }^{{ }^{2} b} c\left\{b, \partial_{3} c\right\}(2)(1), b b^{\prime} b^{-1}
\end{aligned}
$$

elde edilir. Böylece

$$
H_{3}=(C \rtimes B) \rtimes(B \rtimes A) \times(A \rtimes N)
$$

Yarı direk çarpımı elde edilir. Dejenere ve yüz operatörlerimiz

$$
\begin{gathered}
d_{0}:=(C \rtimes B) \rtimes(B \rtimes A) \rtimes(A \rtimes N) \rightarrow(B \rtimes A) \rtimes(A \rtimes N) \\
\left(c, b, b^{\prime}, a, a^{\prime}, n\right) \rightarrow\left(b^{\prime}, a, a^{\prime}, n\right) \\
d_{1}:=(C \rtimes B) \rtimes(B \rtimes A) \rtimes(A \rtimes N) \rightarrow(B \rtimes A) \rtimes(A \rtimes N) \\
\left(c, b, b^{\prime}, a, a^{\prime}, n\right) \rightarrow\left(b, a, a^{\prime}, n\right) \\
d_{2}:=(C \rtimes B) \rtimes(B \rtimes A) \rtimes(A \rtimes N) \rightarrow(B \rtimes A) \rtimes(A \rtimes N) \\
\left(c, b, b^{\prime}, a, a^{\prime}, n\right) \rightarrow\left(b b^{\prime}, a, a^{\prime}, n\right) \\
d_{3}:=(C \rtimes B) \rtimes(B \rtimes A) \rtimes(A \rtimes N) \rightarrow(B \rtimes A) \rtimes(A \rtimes N) \\
\left(c, b, b^{\prime}, a, a^{\prime}, n\right) \rightarrow\left(\partial_{3} c b, \partial_{2} b^{\prime} a, a^{\prime}, n\right) \\
s_{0}:=(B \rtimes A) \rtimes(A \rtimes N) \rightarrow(C \rtimes B) \rtimes(B \rtimes A) \rtimes(A \rtimes N) \\
\left(b, a, a^{\prime}, n\right) \rightarrow\left(1, b, 1, a, a^{\prime}, n\right) \\
s_{1}:=(B \rtimes A) \rtimes(A \rtimes N) \rightarrow(C \rtimes B) \rtimes(B \rtimes A) \rtimes(A \rtimes N) \\
\left(b, a, a^{\prime}, n\right) \rightarrow\left(1,1, b, a, a^{\prime}, n\right)
\end{gathered}
$$

Böylece 3-truncated pseudosimplisel grup $H=\left\{H_{0}, H_{1}, H_{2}, H_{3}\right\}$ elde ederiz.

\section{KAYNAKÇA (REFERENCES)}

[1] J.H.C. Whitehead. Combinatorial Homotopy II, Bull . American Math. Society, 453-456, (1949).

[2] T. Porter. Homology of Commutative Algebras and an Invariant of Simis and Vasconceles. J. Algebra 
99, 458-465(1986).

[3] R. Brown, Higher Dimensional Group Teory, Low Dimensional Topology, London Math. Soc. Lecture Note Series, 48, 215-238 (1982).

[4] R. Brown. Coproducts of Crossed P-modules, Applications to Second Homtopy Groups and to the Hmology of Groups, Topology, 23, 337-345, (1984).

[5] R. Brown and P.J. Higgins. Colmit Theorems for Relative Homotopy Groups, J. Pure Appl. Algebra, 22, 248-370, (1981).

[6] D. Conduch' e, Modules crois'es g'en'eralis'es de longueur 2, Journal of Pure and Applied Algebra, 34, 155-178, (1984).

[7] P. Carrasco and A. M. Cegarra, Group-theoretic Algebraic Models for Homotopy Types, Jour. Pure Applied Algebra, 75, 195-235,(1991)

[8] A. Mutlu and T. Porter, Applications of Peiffer pairing in the Moore complex of a simplicial group, Theory and Applications of Categories, Volume 4, No. 7, 148-173 (1998).

[9] Z. Arvasi, T.S. Kuzpınarı and E. Ö. Uslu, Three Crossed Modules, Homology, Homotopy and Aplications, $11(2), 161-187$.

[10] Inasaridze, H. N.: Homotopy of pseudosimplicial groupsand nonabelian derived functors and algebraic K-theory, Math. Sbornik, TOM, 98, (140), No: 3, 303-323 (1975).

[11] İ. Akça and S. Pak Pseudo simplicial groups and crossed modules, Turk J Math, Volume 34, 475-487 (2010).

[12] U. Cesur, Pseudo Pseudo 3- Çaprazlanmış Modüller, Necmettin Erbakan Üniversitesi Fen Bilimleri Enstitüsü, KONYA (2018)

[13] P. Carrasco, Complejos hipercruzados, cohomologiay extensiones, Ph.D. Thesis, Universidad de Granada, (1987). 